\title{
Parkin on serine: a Parkinson disease gene suppresses serine synthesis in cancer
}

\author{
W. Brian Dalton \\ Department of Oncology, Johns Hopkins University School of Medicine, Baltimore, Maryland, USA
}

\begin{abstract}
Phosphoglycerate dehydrogenase (PHCDH) catalyzes the first step in the synthesis of the amino acid serine, important for protein synthesis, one-carbon metabolism, lipid production, redox homeostasis, and other key processes of normal and cancer metabolism. While PHCDH is often overexpressed in cancer cells, how it is regulated has been unclear. In this issue of the $J C I$, Liu and colleagues describe a new aspect of PHGDH regulation, demonstrating that the Parkinson disease gene and tumor suppressor Parkin bound and ubiquitinated PHCDH. Parkin promoted PHCDH degradation, suppressed serine synthesis, and inhibited tumor growth in human cancer cell line xenografts. Conversely, inactivation of Parkin not only accelerated tumor growth, but also sensitized tumors to small molecule inhibitors of PHCDH. These results offer a new link between Parkin and the serine synthesis pathway, and they bear translational potential that warrants further study in Parkin-deficient human cancers.
\end{abstract}

\section{The serine synthesis pathway}

The reprogramming of cellular metabolism has emerged as a bona fide hallmark of cancer, now comfortably seated next to canonical phenotypes such as replicative immortality and sustained proliferative signaling $(1,2)$. Cancers alter their metabolism for many reasons, including adaptation to limited nutrient conditions, fueling the biosynthetic demands of rapid proliferation, and reprogramming gene expression through metabolites (1). While much is still unknown about the underlying causes of metabolic reprogramming, known mechanisms include direct mutation of metabolic enzymes (i.e., isocitrate dehydrogenase 1 and 2, fumarate dehydratase, succinate dehydrogenase) and indirect effects of mutations in pleiotropic cancer genes (i.e., KRAS, MYC, NRF2) (3). These changes also create metabolic vulnerabilities that can be therapeutically targeted, such as with longstanding ther- apies, such as L-asparaginase, recent arrivals, such as inhibitors of mutant isocitrate dehydrogenase $1 / 2$, and many investigational therapies, such as glutaminase inhibitors and arginine-depleting enzymes (3). For these reasons, identifying mechanisms of metabolic reprogramming in cancer promises to advance both understanding of disease biology and development of metabolic therapies.

One metabolic process commonly reprogrammed in cancer is the serine synthesis pathway (SSP). Initiated by the rate-limiting conversion of the glycolytic intermediate 3-phosphoglycerate to 3-phosphohydroxypyruvate by the enzyme phosphoglycerate dehydrogenase (PHG$\mathrm{DH})$, this pathway produces the nonessential amino acid serine, important in protein synthesis, one-carbon metabolism, lipid production, redox homeostasis, and other vital cellular processes. In cancer, PHG$\mathrm{DH}$ expression is frequently elevated, and
Related Article: p. 3253

Conflict of interest: WBD receives research support from Eli Lilly and AbbVie and has patent US2018/044553 pending. Copyright: @ 2020, American Society for Clinical Investigation.

Reference information: / Clin Invest. 2020;130(6):2820-2822. https://doi.org/10.1172/JCI137411. serine synthesis is frequently increased (4). Elegant studies in mice have shown that upregulation of the SSP is selected by tumors to gain a growth advantage in tissues where extracellular serine is limiting (5). In some cancers, this occurs through amplification of the PHGDH gene $(6,7)$. In others, it may be transcriptional upregulation of PHGDH produced by alterations in genes such as MYC and NRF2 $(8,9)$. However, in most cancers, the mechanisms behind reprogramming of the SSP are unknown. Discovering these mechanisms may have therapeutic, as well as biologic, value. Several small molecule inhibitors of PHGDH have been developed, and they show preclinical anticancer activity in tumors with upregulation of the SSP (10-12).

\section{A mutated ubiquitin ligase}

In this issue of the JCI, Liu et al. (13) report a unique mechanism of $\mathrm{PHGDH}$ regulation by the Parkin (or PRKN) gene, a ubiquitin ligase that is mutated in a tumorsuppressor pattern in several cancer types (Figure 1). The authors started with a mass spectrometry screen of PHGDH-binding partners and found that the IBR domain of Parkin bound the SBD2 domain of PHG$\mathrm{DH}$. This interaction was accompanied by ubiquitination of PHGDH at lysine 330 by Parkin, and this ubiquitination catalyzed the degradation of PHGDH in human cancer cell lines. The researchers then showed that degradation of PHGDH protein by Parkin suppressed serine synthesis, inhibited cell proliferation, and restrained growth of human cancer cell lines as xenografts in immunodeficient mice. Finally, the authors reported that human cancer cell lines with knockout of Parkin exhibited increased sensitivity to small molecule inhibitors of PHGDH, including the bioavailable compound NCT-503, which preferentially inhibited the growth of Parkin-knockout tumors in vivo (13).

Liu et al. (13) make several interesting contributions to our understanding of cancer metabolism. First, the authors 
A
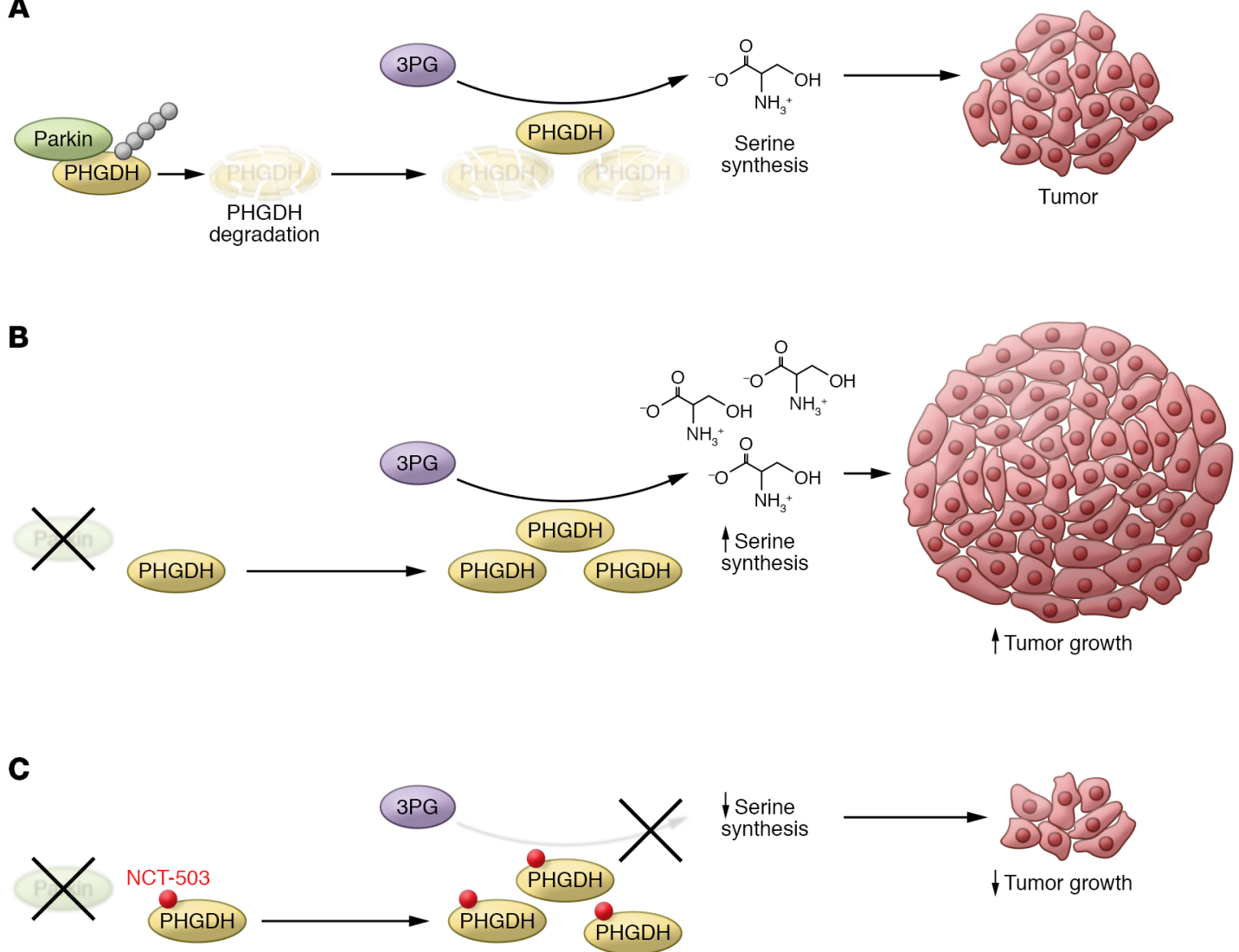

Figure 1. Proposed mechanism for how Parkin suppresses serine synthesis and tumor growth. (A) Tumors with intact Parkin target PHCDH for degradation, restricting intracellular serine synthesis and tumor growth. (B) Tumors lacking Parkin (due to knockout) have elevated PHGDH, serine synthesis, and tumor growth. (C) Targeting PHGDH with the small molecule NCT-503 inhibits serine synthesis and growth of Parkin knockout tumors.

identify a unique mode of SSP regulation through the ubiquitination of PHGDH. A previous study reported that PHGDH protein levels are positively regulated by the deubiquinating enzyme JOSD2, suggesting that differential activity of this enzyme may account for different levels of PHGDH protein in lung adenocarcinoma (14). The present work (13) reveals the opposing regulation - ubiquitination - expanding the known toolkit by which cells can regulate the SSP and by which cancer cells can disrupt it. It will be interesting for future studies to determine the extent to which cancers actually use this mechanism to upregulate the SSP. Moreover, given recent literature showing upregulation of the SSP upon acquired resistance to different cancer therapies, it would be interesting to determine whether higher PHGDH ever results from adaptive changes in JOSD2 or Parkin activity in these contexts (15-17).

Second, Liu et al. (13) identify a hitherto unknown oncogenic property of Parkin, a tumor-suppressor gene whose downstream mediators of oncogenesis are not well understood (13). Reprogramming of serine metabolism now joins other processes whose disruption by Parkin may be tumorigenic, such as cell cycle regulation, apoptosis, and mitophagy $(18,19)$. Inactivating mutations in Parkin are recurrent in a variety of human tumors, and inactivation of Parkin promotes tumor formation in genetically engineered mouse models (GEMMs) (20-22). While the authors focused here on human cancer cell line models, it will be interesting for future studies to determine the role that modulating serine synthesis has in the tumor properties of patient-derived xenografts (PDXs) with naturally arising Parkin mutations - and in GEMMs.

Third, in finding that cancer cell lines with Parkin knockout show increased sensitivity to PHGDH inhibition in vivo, Liu et al. (13) provide evidence that metabolic rewiring by Parkin downregulation forces a targetable dependence on the SSP. By extension, dependence on the SSP suggests that Parkin mutation status could provide a biomarker for tumors to be targeted in any eventual clinical trials of inhibitors of the SSP. As above with tumorigenicity, however, it would be important to first determine whether PHGDH inhibitors show activity against PDX models of human tumors with naturally occurring Parkin mutations. In addition, recent evidence shows that proliferation of cells with an upregulated SSP are constrained by availability of the electron acceptor $\mathrm{NAD}^{+}$, conferring a heightened sensitivity to small molecule inhibitors of the $\mathrm{NAD}^{+}$ salvage pathway enzyme NAMPT $(23,24)$. It could therefore be of interest to determine whether tumors with Parkin mutation are likewise sensitive to these inhibitors.

Finally, as Parkin mutations are causatively implicated in cases of autosomal recessive juvenile Parkinson disease (PD), the authors note that it is possible that deregulation of the SSP might have a role in PD pathophysiology (25). 


\section{Clinical implications}

In describing a metabolic vulnerability to inhibition of PHGDH in human cancer cells with Parkin inactivation, Liu et al. identify a potential biomarker that could be used in future clinical trials of therapies that target the SSP in cancer patients.

Address correspondence to: W. Brian Dalton, Sidney Kimmel Comprehensive Cancer Center at Johns Hopkins, 1650 Orleans Street, CRBI Room 285, Baltimore, Maryland 21231, USA. Phone: 410.614.6173; Email:wdalton2@jhmi.edu.

1. Pavlova NN, Thompson CB. The emerging hallmarks of cancer metabolism. Cell Metab. 2016;23(1):27-47.

2. Hanahan D, Weinberg RA. Hallmarks of cancer: the next generation. Cell. 2011;144(5):646-674.

3. Vander Heiden MG, DeBerardinis RJ. Understanding the intersections between metabolism and cancer biology. Cell. 2017;168(4):657-669.

4. Mattaini KR, Sullivan MR, Vander Heiden MG. The importance of serine metabolism in cancer. J Cell Biol. 2016;214(3):249-257.

5. Sullivan MR, et al. Increased serine synthesis provides an advantage for tumors arising in tissues where serine levels are limiting. Cell Metab. 2019;29(6):1410-1421.e4.

6. Possemato R, et al. Functional genomics reveal that the serine synthesis pathway is essential in breast cancer. Nature. 2011;476(7360):346-350.

7. Locasale JW, et al. Phosphoglycerate dehydrogenase diverts glycolytic flux and contributes to oncogenesis. Nat Genet. 2011;43(9):869-874.

8. DeNicola GM, et al. NRF2 regulates serine biosynthesis in non-small cell lung cancer. Nat Genet. 2015;47(12):1475-1481.

9. Nilsson LM, et al. Mouse genetics suggests cell-context dependency for Myc-regulated metabolic enzymes during tumorigenesis. PLoS Genet. 2012;8(3):e1002573.

10. Pacold ME, et al. A PHGDH inhibitor reveals coordination of serine synthesis and one-carbon unit fate. Nat Chem Biol. 2016;12(6):452-458.

11. Mullarky E, et al. Identification of a small molecule inhibitor of 3-phosphoglycerate dehydrogenase to target serine biosynthesis in cancers. Proc Natl Acad Sci USA. 2016;113(7):1778-1783.

12. Wang $\mathrm{Q}$, et al. Rational design of selective allosteric inhibitors of PHGDH and serine synthesis with anti-tumor activity. Cell Chem Biol. 2017;24(1):55-65.

13. Liu J,et al. Parkin ubiquitinates phosphoglycerate dehydrogenase to suppress serine synthesis and tumor progression. J Clin Invest. 2020;130(6):3253-3269.

14. Zhang B, et al. PHGDH defines a metabolic subtype in lung adenocarcinomas with poor prognosis. Cell Rep. 2017;19(11):2289-2303.

15. Wei L, et al. Genome-wide CRISPR/Cas9 library screening identified $\mathrm{PHGDH}$ as a critical driver for Sorafenib resistance in HCC. Nat Commun. 2019;10(1):4681.

16. Wu X, et al. Phosphoglycerate dehydrogenase promotes proliferation and bortezomib resis- tance through increasing reduced glutathione synthesis in multiple myeloma [published online February 10, 2020]. Brit J Haematol. https://doi. org/10.1111/bjh.16503.

17. Zaal EA, Wu W, Jansen G, Zweegman S, Cloos J, Berkers CR. Bortezomib resistance in multiple myeloma is associated with increased serine synthesis. Cancer Metab. 2017;5:7.

18. Gong Y, et al. Pan-cancer genetic analysis identifies PARK2 as a master regulator of G1/S cyclins. Nat Genet. 2014;46(6):588-594.

19. Liu J, Zhang C, Hu W, Feng Z. Parkinson's disease-associated protein Parkin: an unusual player in cancer. Cancer Commun (Lond). 2018;38(1):40.

20. Poulogiannis G, et al. PARK2 deletions occur frequently in sporadic colorectal cancer and accelerate adenoma development in Apc mutant mice. Proc Natl Acad Sci USA. 2010;107(34):15145-15150.

21. Fujiwara M, et al. Parkin as a tumor suppressor gene for hepatocellular carcinoma. Oncogene. 2008;27(46):6002-6011.

22. Veeriah S, et al. Somatic mutations of the Parkinson's disease-associated gene PARK2 in glioblastoma and other human malignancies. Nat Genet. 2010;42(1):77-82.

23. Murphy JP, et al. The $\mathrm{NAD}^{+}$salvage pathway supports PHGDH-driven serine biosynthesis. Cell Rep. 2018;24(9):2381-2391.e5.

24. Diehl FF, Lewis CA, Fiske BP, Vander Heiden MG. Cellular redox state constrains serine synthesis and nucleotide production to impact cell proliferation. Nat Metab. 2019;1(9):861-867.

25. Arkinson C, Walden H. Parkin function in Parkinson's disease. Science. 2018;360(6386):267-268. 